\title{
Customising Web Information Systems According to User Preferences
}

\author{
Klaus-Dieter Schewe • Bernhard Thalheim • \\ Qing Wang
}

Received: 12 May 2008 / Revised: 1 October 2008 /

Accepted: 3 November 2008 / Published online: 12 November 2008

(C) Springer Science + Business Media, LLC 2008

\begin{abstract}
Web Information Systems have to serve a variety of users with very diverse preferences regarding content, functionality and presentation. We first investigate the customisation of functionality at a high-level of abstraction, where possible action sequences are represented by an algebraic expression called plot, and user preferences give rise to equations. We show that the problem can be solved by applying conditional term rewriting on the basis of Kleene algebras with tests. By exploiting the idea of weakest preconditions such expressions can be represented by formal power series with coefficients in a Boolean algebra. This gives rise to a sufficient condition for termination based on well-founded orders on such power series. As confluence cannot be guaranteed, we propose critical pair completion to be used in order to enforce the desirable Church-Rosser property. In a second step we parametrise the actions and replace the Boolean conditions by first-order formulae. We show that still term rewriting can be applied, but termination and Church Rosser property become problems that will require manual interaction, in particular, as preference rules will make use of the parameters. On the other hand the presence of first-order conditions can be used to extend the customisation to the content.
\end{abstract}

Keywords web information system • personalisation • term rewriting • Kleene algebra with tests $\cdot$ Church-Rosser property $\cdot$ termination • critical pair completion $\cdot$ media type $\cdot$ deontic constraint $\cdot$ preference rule

K.-D. Schewe $(\bowtie) \cdot$ Q. Wang

Information Science Research Centre, Palmerston North, New Zealand e-mail: kdschewe@acm.org

Q. Wang

e-mail: wang0qing@gmail.com

B. Thalheim

Department of Computer Science, Christian Albrechts University Kiel, Olshausenstr. 40, D-24098 Kiel, Germany e-mail: thalheim@is.informatik.uni-kiel.de 


\section{Introduction}

A Web Information System (WIS) is an Information System that is accessible via the world-wide web. As such it is open to a variety of users, who all have preferences regarding content, functionality and presentation. The more a WIS respects the personal preferences of its users on how to use the system, the higher are its chances of being accepted. Quite often, however, the major emphasis of WIS personalisation is the presentation aspect. Our major concern here is the functionality in the sense that we want to discard actions from a WIS specification, if the user preferences imply that they will not be executed, but we will also devote some initial thoughts to the customisation of content.

In order to address the customisation of WISs according to user preferences we adopt the co-design approach to WIS development [22], because it offers the possibility to look at a WIS on different levels of abstraction, and these levels are well integrated. This does not exclude our approach from being used also in combination of other WIS engineering methods, e.g. WISM [7], HERA [10], WebML [4], and UML-based models $[5,16]$. It may also be used for the restricted class of formoriented systems [8].

On a high-level of abstraction a Web Information System (WIS) can be represented by a storyboard, which in an abstract way captures who will be using the system, in which way and for which goals. An important component of a storyboard is the plot that is specified by an assignment-free process, thus captures the possible action sequences, each of which is called a story. The formalisation of plots has been addressed in [1] using ASMs and in [20,22] using Kleene Algebras with Tests (KATs) [13]. In both cases the motivation for the formalisation was the ability to formally reason about a WIS specification already at a high-level of abstraction in order to capture the anticipated usage of the system.

Another important component of a storyboard is given by the actors, which among others comprise user roles and user profiles [22]. A role prescribes obligations and rights a user in that role has. This can be expressed by propositional deontic logic constraints. A profile characterises the properties of a user leading to preference rules. We do not discuss the specification of profiles in this paper, as for our purpose here it is sufficient to have an understanding of preference rules.

The formalisation of a plot by an algebraic expression can be used to apply term rewriting on the basis of equations that may be used to express user preferences. In this way the research contributes to the important personalisation problem for WISs using an inferential approach rather than duplicating its specification. This idea was further investigated in [21] and [23]. In [24] we established conditions, under which term rewriting will terminate and produce a unique result, i.e. it can be effectively applied.

A system of equations on a KAT defines a quotient in a natural way. What we are interested in are "simple" representatives of equivalence classes in the case of KATs that are generated out of some finite set of atoms. We derive a canonical representation through formal power series with coefficients in a Boolean algebra, which arises by adopting weakest preconditions. This is the basis for the definition of power series orders on KATs, which will allow us to characterize effective systems of equations by sufficient conditions for terminating term rewriting. In order to 
circumvent the confluence problems we adopt Critical Pair Completion (CPC) as the key element of the Knuth-Bendix algorithm [12].

As emphasised in our previous work it would be desirable to shift the term rewriting approach to WIS customisation from the propositional high-level of abstraction to the conceptual level, on which we actually deal with databases and operations that update them. As outlined in [19] this would lead naturally to a higher-order dynamic logic, and very little is known about inferences in such a complex logic. Therefore, in this paper we will only make a small step in the direction of WIS customisation on the conceptual level by using parameterised plots, an idea that has already been used in the context of bargaining problems and their formalisation by means of games on the (parameterised) plot [17].

In particular, the Boolean conditions in the plot can be replaced by first-order formulae, which will allow us to express preferences in much more detail as e.g. in [18] without giving up the possibility to apply term rewriting for simplifying the plot. However, in this way we do not simplify the operations as such, but it is argueable whether such an optimisation of the operations is needed at all.

Furthermore, according to [22] the parameterised operations that refine the actions in the story space are associated with views on the underlying database, and the conditions appearing in the parametrised plot will thus be conditions that are to be evaluated against the underlying database. This may be used to restrict the views themselves thereby leading to a customisation of the content. We will outline this idea, though it is still in a preliminary stage.

The remainder of the paper is organised as follows. In Section 2 we address the high-level customisation of WIS plots according to equational preference rules. This covers more or less our previous work in $[23,24]$, in particular the results on termination and Church Rosser property, but we add a discussion of the compatibility of preference rules with propositional deontic constraints. We show that the deontic constraints give also rise to equations and thus can be handled in the same way with the difference that these equations do not just express preferences, but strict restrictions. In Section 3 we deal with parameterised plots. We first show how the term rewriting approach generalises to this more general setting and how the problems of termination and Church Rosser property can be approached. Then we discuss the customisation of content. We conclude with a brief summary and outlook in Section 4.

\section{Personalisation of plots by term rewriting}

The high-level specification of a WIS by means of a storyboard is an open invitation for personalisation with respect to functionality, whereas personalisation with respect to content or presentation requires more details. Therefore, we concentrate on the plot that describes the possible sequence of actions [22].

\subsection{Plots and Kleene algebras with tests}

In order to formalise plots we refer to Kleene algebras with tests (KATs) [22] that are known to provide a formalism that exceeds the expressiveness of propositional 
Hoare logic [14]. While Kleene algebras are known since the cradle days of automata theory - they were introduced by Kleene in [11] — KATs are a more recent extension. Formally, we obtain the following definition.

Definition 1 A Kleene algebra $(\mathrm{KA}) \mathcal{K}=\left(K,+, \cdot,{ }^{*}, 0,1\right)$ consists of

- a carrier-set $K$ containing at least two different elements 0 and 1 , and

- a unary operation * and two binary operations + and · on $K$

such that the following axioms are satisfied (adopting the convention to write $p q$ for $p \cdot q$, and to assume that $\cdot$ binds stronger than +$)$ :

- $\quad+$ and $\cdot$ are associative, i.e. for all $p, q, r \in K$ we must have $p+(q+r)=$ $(p+q)+r$ and $p(q r)=(p q) r$

- $\quad+$ is commutative and idempotent with 0 as neutral element, i.e. for all $p, q \in K$ we must have $p+q=q+p, p+p=p$ and $p+0=p$;

- $\quad 1$ is a neutral element for $\cdot$, i.e. for all $p \in K$ we must have $p 1=1 p=p$;

- for all $p \in K$ we have $p 0=0 p=0$;

- $\quad$ is distributive over + , i.e. for all $p, q, r \in K$ we must have $p(q+r)=p q+p r$ and $(p+q) r=p r+q r$

- $\quad p^{*} q$ is the least solution $x$ of $q+p x \leq x$ and $q p^{*}$ is the least solution of $q+x p \leq$ $x$, using the partial order $x \leq y \equiv x+y=y$.

Definition 2 A Kleene algebra with tests $(\mathrm{KAT}) \mathcal{K}=\left(K, B,+, \cdot, *_{,}^{-}, 0,1\right)$ consists of

- $\quad$ a Kleene algebra $\left(K,+, \cdot,{ }^{*}, 0,1\right)$;

- a subset $B \subseteq K$ containing 0 and 1 and closed under + and $\cdot$;

- $\quad$ and a unary operation ${ }^{-}$on $B$, such that $(B,+, \cdot,, 0,1)$ forms a Boolean algebra.

Example 1 Take a simple example, where the WIS is used for ordering products, which we adopt from [23]. In this case we may define four scenes:

- $\quad$ The scene $s_{0}=$ product would contain product descriptions and allow the user to select products.

- $\quad$ The scene $s_{1}=$ payment will be used to inform the user about payment method options and allow the user to select the appropriate payment method.

- The scene $s_{2}=$ address will be used to require information about the shipping address from the user.

- Finally, scene $s_{3}=$ confirmation will be used to get the user to confirm the order and the payment and shipping details.

There are six actions (their names are sufficient to indicate what they are supposed to do)

- $\quad \alpha_{1}=$ select_product is defined on $s_{0}$ and leads to a transition to scene $s_{1}$.

- $\quad \alpha_{2}=$ payment_by_card is defined on $s_{1}$ and leads to a transition to scene $s_{2}$.

- $\quad \alpha_{3}=$ payment_by_bank_transfer is defined on $s_{1}$ and leads to a transition to scene $s_{2}$.

- $\quad \alpha_{4}=$ payment_by_cheque is defined on $s_{1}$ and leads to a transition to scene $s_{2}$.

- $\quad \alpha_{5}=$ enter_address is defined on $s_{2}$ and leads to a transition to scene $s_{3}$.

- $\quad \alpha_{6}=$ confirm_order is defined on $s_{3}$ and leads to a transition to scene $s_{0}$. 
and five Boolean conditions:

- The condition $\varphi_{1}=$ price_in_range expresses that the price of the selected product(s) lies within the range of acceptance of credit card payment. It is a precondition for action $\alpha_{2}$.

- The condition $\varphi_{2}=$ payment_by_credit_card expresses that the user has selected the option to pay by credit card.

- The condition $\varphi_{3}=$ payment_by_bank_transfer expresses that the user has selected the option to pay by bank transfer.

- $\quad$ The condition $\varphi_{4}=$ payment_by_cheque expresses that the user has selected the option to pay by cheque.

- The condition $\varphi_{5}=$ order_confirmed expresses that the user has confirmed the order.

Using these actions and conditions, we can formalise the story space by the algebraic expression $\left(\alpha_{1}\left(\varphi_{1} \alpha_{2} \varphi_{2}+\alpha_{3} \varphi_{3}+\alpha_{4} \varphi_{4}\right) \alpha_{5}\left(\alpha_{6} \varphi_{5}+1\right)+1\right)^{*}$.

\subsection{Quotient Kleene algebras with tests}

As already stated in the introduction, we are interested in equations on KATs. Taking an interpretation by assignment-free process involving propositional conditions as in [20], some equations can be interpreted as user preferences-a rule of thumb is that the equation must restrict choices - and general knowledge about the application area. In particular, equations of the following form are of interest:

- If a process $p$ has a precondition $\varphi$, then we obtain the equation $\bar{\varphi} p=0$.

- If a process $p$ has a postcondition $\psi$, we obtain the equation $p=p \psi$ or equivalently $p \bar{\psi}=0$.

- An equation $\varphi\left(p_{1}+p_{2}\right)=\varphi p_{1}$ expresses a conditional preference of $p_{1}$ over $p_{2}$ in case the condition $\varphi$ is satisfied.

- An equation $p\left(p_{1}+p_{2}\right)=p p_{1}$ expresses another conditional preference of $p_{1}$ over $p_{2}$ after the process $p$.

- An equation $p_{1} p_{2}+p_{2} p_{1}=p_{1} p_{2}$ expresses a preference of order.

Thus, let $\Sigma$ denote a set of equations on a $\operatorname{KAT} \mathcal{K}=\left(K, B,+, \cdot, *_{,}^{-}, 0,1\right)$. Then $\Sigma$ defines an equivalence relation $\sim$ on $K$ in the usual way, i.e. $p \sim q$ holds iff $p=q$ is implied by $\Sigma$ and the axioms for KATs in Definitions 1 and 2.

Let $K / \Sigma$ denote the set of equivalence classes $[p]$ under $\sim$ with $p \in K$, and $B / \Sigma$ the subset $\{[b] \mid b \in B\}$. Define the KAT operations on $K / \Sigma$ in the natural way, i.e. $[p]+[q]=[p+q],[p][q]=[p q],[p]^{*}=\left[p^{*}\right], 0=[0]$, and $1=[1]$, and the complementation on $B / \Sigma$ by $\overline{[b]}=[\bar{b}]$.

Proposition 1 If $\mathcal{K}=\left(K, B,+, \cdot,{ }^{-},, 0,1\right)$ is a $K A T$ and $\Sigma$ a set of equations on $K$, then the quotient $\mathcal{K} / \Sigma=\left(K / \Sigma, B / \Sigma,+, \cdot, *,{ }^{-}, 0,1\right)$ is a $K A T$.

Proof We have to verify the axioms of Kleene algebras in Definitions 1 and 2. As equivalence $p \sim q$ is defined by the derivability of the equation $p=q$, we can simply replace $p$ by $q$ in any equation. 


\subsection{Term rewriting with KAT expressions}

At the level of plots we can address personalisation with respect to functionality, i.e. we restrict the plot according to user preferences and goals. As this leads to equations as outlined above, we are actually looking for a simplified KAT expression $p^{\prime}$ for a plot $p$ such that $p$ and $p^{\prime}$ represent the same element in $\mathcal{K} / \Sigma$. In [23] we suggested to use term rewriting for this. As standard in order-sorted algebraic specifications we take two sorts $B$ and $K$ ordered by $B \leq K$, and the following (nullary, unary, binary) operators:

$$
0,1: \rightarrow B \quad+, \cdot: K K \rightarrow K^{*}: K \rightarrow K^{-}: B \rightarrow B
$$

Using these sorts and operators we can define terms in the usual way.

A rewrite rule is an expression of the form $\lambda \rightsquigarrow \varrho$ with terms $\lambda$ and $\varrho$ of the same sort, such that the variables on the right hand side $\varrho$ are a subset of the variables on the left hand side $\lambda$. A conditional rewrite rule is an expression of the form $t_{1}=t_{2} \rightarrow$ $\lambda \rightsquigarrow \varrho$, in which in addition the terms $t_{1}$ and $t_{2}$ contain the same variables and these form a superset of the set of variables in the left hand side term $\lambda$.

The application of a rewrite rule $\lambda \rightsquigarrow \varrho$ to a term $t$ is standard: if $t$ contains a subterm $t^{\prime}$ that can be matched with $\lambda$, i.e. there is a substitution $\theta$ such that the application of $\theta$ to $\lambda$ results in $t^{\prime}$ (denoted $\theta \cdot \lambda=t^{\prime}$ ), then replace $t^{\prime}$ in $t$ by $\theta$. $\varrho$.

The application of a conditional rewrite rule $t_{1}=t_{2} \rightarrow \lambda \rightsquigarrow \varrho$ to a term $t$ is defined analogously. Precisely, if $t$ contains a subterm $t^{\prime}$ that can be matched with $\lambda$, i.e. there is a substitution $\theta$ such that the application of $\theta$ to $\lambda$ results in $t^{\prime}$ (denoted $\theta \cdot \lambda=$ $\left.t^{\prime}\right)$, then replace $t^{\prime}$ in $t$ by $\theta . \varrho$. However, in this case we have to show that $\theta . t_{1}=$ $\theta . t_{2}$ holds for the substitution $\theta$. For this we start a separate term-rewriting process that aims at showing $\theta . t_{1} \rightsquigarrow \cdots \rightsquigarrow \theta . t_{2}$. We call this separate rewriting process a coroutine, because we can run it in parallel to the main rewriting process. The risk is of course that if we fail to verify $\theta . t_{1}=\theta . t_{2}$, then we have to backtrack to $t$ for the main rewriting process.

In order to exploit term-rewriting for the personalisation problem we formulate the axioms of KATs and the personalisation equations as (conditional) rewrite rules, then start with $p \psi$ and apply the rules until we finally obtain a term of the form $p^{\prime} \psi$ to which no more rule can be applied. Note that $p \psi$ is closed, i.e. it does not contain variables, so during the whole rewriting process we will only have to deal with closed terms.

We use $p, q, r, \ldots$ (if needed with additional indices) as variables of sort $K$, and $a, b, c, \ldots$ (also with indices) as variables of sort $B$. Then we use the following general (conditional) rewrite rules:

$$
\begin{array}{rlrl}
p+(q+r) & \rightsquigarrow(p+q)+r & p(q r) & \rightsquigarrow(p q) r \\
p+p & \rightsquigarrow p & p+0 & \rightsquigarrow p \\
p 1 & \rightsquigarrow p & 1 p & \rightsquigarrow p \\
p 0 & \rightsquigarrow 0 & (p+q) r & \rightsquigarrow p r+q r \\
p(q+r) & \rightsquigarrow p q+p r & 1+p^{*} p & \rightsquigarrow p^{*}
\end{array}
$$




$$
\begin{array}{rlrl}
p q+q=q \rightarrow p^{*} q+q & \rightsquigarrow q & q p+q=q \rightarrow q p^{*}+q & \rightsquigarrow q \\
p+q & \rightsquigarrow q+p & a b & \rightsquigarrow b a \\
a \bar{a} & \rightsquigarrow 0 & \bar{a} a & \rightsquigarrow 0 \\
a+\bar{a} & \rightsquigarrow 1 & \bar{a}+a & \rightsquigarrow 1
\end{array}
$$

As an extension we apply these rewriting rules $\lambda \rightsquigarrow \varrho$ not only in one direction, but we also use the reverse rules $\varrho \rightsquigarrow \lambda$ (similar for conditional rewrite rules). In addition, the personalisation equations from above give rise to further rewrite rules:

- A conditional preference equation gives rise to a rule of the form $a(p+q) \rightsquigarrow a p$.

- A precondition gives rise to a rule of the form $\bar{a} p \rightsquigarrow 0$.

- A postcondition gives rise to a rule of the form $p \bar{a} \rightsquigarrow 0$.

- An invariance condition gives rise to a rule of the form $a p \bar{a}+\bar{a} p a \rightsquigarrow 0$.

- An exclusion condition gives rise to a rule of the form $a b \rightsquigarrow 0$.

Example 2 Let us continue Example 1 and localise the plot, i.e. personalise with respect to the location of its users. Consider a European user, for which the option to pay by cheque should be invalidated. So we obtain the equation $\alpha_{4}=0$. Furthermore, assume that this user prefers to pay by credit card, if this turns out to be a valid option. For this we use the equation $\varphi_{1}\left(\alpha_{3}+\alpha_{4}\right)=0$, which also implies $\varphi_{1} \alpha_{3}=0$. Let again $\psi=1$. Now we compute

$$
\begin{aligned}
p \psi & =\left(\alpha_{1}\left(\varphi_{1} \alpha_{2} \varphi_{2}+\alpha_{3} \varphi_{3}+\alpha_{4} \varphi_{4}\right) \alpha_{5}\left(\alpha_{6} \varphi_{5}+1\right)+1\right)^{*} \\
& \rightsquigarrow\left(\alpha_{1}\left(\varphi_{1} \alpha_{2} \varphi_{2}+\alpha_{3} \varphi_{3}\right) \alpha_{5}\left(\alpha_{6} \varphi_{5}+1\right)+1\right)^{*} \\
& \rightsquigarrow\left(\alpha_{1}\left(\varphi_{1} \alpha_{2} \varphi_{2}+\left(\varphi_{1}+\bar{\varphi}_{1}\right) \alpha_{3} \varphi_{3}\right) \alpha_{5}\left(\alpha_{6} \varphi_{5}+1\right)+1\right)^{*} \\
& \rightsquigarrow\left(\alpha_{1}\left(\varphi_{1} \alpha_{2} \varphi_{2}+\varphi_{1} \alpha_{3} \varphi_{3}+\bar{\varphi}_{1} \alpha_{3} \varphi_{3}\right) \alpha_{5}\left(\alpha_{6} \varphi_{5}+1\right)+1\right)^{*} \\
& \rightsquigarrow\left(\alpha_{1}\left(\varphi_{1} \alpha_{2} \varphi_{2}+0+\bar{\varphi}_{1} \alpha_{3} \varphi_{3}\right) \alpha_{5}\left(\alpha_{6} \varphi_{5}+1\right)+1\right)^{*} \\
& \rightsquigarrow\left(\alpha_{1}\left(\varphi_{1} \alpha_{2} \varphi_{2}+\bar{\varphi}_{1} \alpha_{3} \varphi_{3}\right) \alpha_{5}\left(\alpha_{6} \varphi_{5}+1\right)+1\right)^{*} \\
& =p^{\prime} \psi,
\end{aligned}
$$

i.e. the option to pay by cheque has been removed from the resulting plot $p^{\prime}$, and the option to pay by bank transfer has become subject to the condition $\bar{\varphi}_{1}$, i.e. the price must be out of range for credit card payment.

\subsection{Formal power series}

The outlined term rewriting process is indeed highly non-deterministic, but we wish to obtain a unique final result. For this we first have to ensure that the process terminates. If this can be done, then the second problem is to ensure confluence, i.e. guaranteeing that the result is independent from the choice of rewrite rules and their order. We will investigate these two problems in this section.

In [20] the idea was launched to approach personalisation by term rewriting on KATs. Indeed, the plot of a storyboard can be easily written as a KAT expression, and preference rules give rise to equations as exemplified in the previous section. 
Then the problem is to find a "simplest" representative $p^{\prime} \in K$ of an equivalence class $[p] \in K / \Sigma$.

Equations like the ones shown in the previous section show indeed a preferred direction. We would rather see the left hand side replaced by the right hand side than the other way round. That is, we could use equations as rewrite rules $\ell \rightsquigarrow r$. This was exemplified in [20, 22] and further elaborated in [23]. It may still be necessary to use rules such as $1 \rightsquigarrow b+\bar{b}$ for some $b \in B$, but for the moment we are primarily interested in termination.

For this we take up the old idea from [6] to define a well-founded order on terms in such a way that the left hand side of the rewrite rules will always be larger than the right hand side. In particular, we will look at an order defined by formal power series. Thus, our first step will be to look for ways to associate a formal power series with the expressions in a $\mathrm{KAT} \mathcal{K}=\left(K, B,+, \cdot, *,{ }^{-}, 0,1\right)$.

In order to so, we start with the assumption that we are given a set $A=$ $\left\{p_{1}, \ldots, p_{k}\right\}$ of atomic processes and a set $P=\left\{b_{1}, \ldots, b_{\ell}\right\}$ of propositional atoms with $A \cap P=\emptyset$. Let $B$ be generated out of $P \cup\{0,1\}$ using conjunction $\cdot$, disjunction + , and negation - Then let $K$ be generated out of $A \cup B \cup\{0,1\}$ using choice +, sequence $\cdot$, and iteration *. More precisely, we have to consider $K / \Sigma$ with $\Sigma$ being the KAT axioms from Definitions 1 and 2, but we ignore this little subtlety.

Note that we actually restrict our attention to finitely generated KATs, which will help us to circumvent the undecidability and complexity problems associated with KATs in general [15].

We even extend the Boolean algebra $B$ by enlarging its base $P$. The idea is that for $p \in A$ and $b \in P \cup\{0\}$ we add the weakest preconditions [ $p] b$ to $P$. The intended meaning is if $p$ starts in a state satisfying $[p] b$, for which it is defined and terminating, then the resulting state will satisfy $b$. Formally, $[p] b$ is the largest solution $x$ of $x p \bar{b}=0$. For simplicity let us stay with the notation $P, B$ and $K$.

Definition 3 A monom over $A$ of degree $m$ is an expression of the form $q_{1} \ldots q_{m}$ with all $q_{i} \in A$. A formal power series over $A$ and $P$ is an expression of the form

$$
\sum_{i=0}^{\infty} \sum_{\sigma:\{1, \ldots, i\} \rightarrow\{1, \ldots, k\}} b_{\sigma(1) \ldots \sigma(i)} p_{\sigma(1)} \ldots p_{\sigma(i)}
$$

with coefficients $b_{\sigma(1) \ldots \sigma(i)} \in B$. Let $\mathcal{F P \mathcal { S }}(K)$ denote the set of formal power series over $K$.

Note that the Boolean algebra $B$ in this definition contains all the preconditions, and the $p_{\sigma(1)} \ldots p_{\sigma(i)}$ are indeed monoms of degree $i$. Furthermore, assuming an order $\leq$ on $A$, this can be canonically extended to a total order on monoms, in which monoms of lower degree precede all those of higher degree.

We have to ensure that these basic preconditions are sufficient. For this we shift Boolean conditions in front of processes leading to the following proposition.

Proposition 2 With the definitions above each $K A T$ expression $p \in K$ can be represented by a formal power series $\llbracket p \rrbracket \in \mathcal{F} \mathcal{P} \mathcal{S}(K)$. 
Proof Looking at composed propositions we have

$$
[p]\left(b_{1} b_{2}\right)=\left([p] b_{1}\right)\left([p] b_{2}\right) \quad \text { and } \quad[p] \bar{b}=\overline{[p] 0[p] b},
$$

which together with $b_{1}+b_{2}={\overline{b_{1}}}_{1} \bar{b}_{2}$ allows us to replace composed propositions by simply ones in $P$.

For complex processes in $K$ we obtain $[1] b=b,[0] b=0,[p q] b=[p][q] b$, and $[p+q] b=([p] b)([q] b)$. In addition, $\left[p^{*}\right] b$ is the smallest solution $x$ of $x=b[p] x$, but this may not be needed, as we can use the formal power series $p^{*}=1+p+$ $p^{2}+\cdots=\sum_{i=0}^{\infty} p^{i}$ for our purposes, which will result in

$$
\left[p^{*}\right] b=\sum_{i=0}^{\infty}\left[p^{i}\right] b=\sum_{i=0}^{\infty} \underbrace{[p] \ldots[p]}_{i \text { times }} b .
$$

According to the definition of weakest precondition we have $[p] b p=p b$, so we obtain that all propositional conditions can be shifted to the front.

\subsection{Termination}

The second step towards our goal of terminating term rewriting consists of defining a well-founded partial order on $K$ such that for rewrite rules $\ell \rightsquigarrow r$ we are interested in we obtain $\ell \geq r$. This suffices to guarantee termination [6]. We will concentrate on fps orders.

Definition 4 An fps order $\leq$ on $K$ is defined by a valuation $v: B \rightarrow \mathbb{R}$.

For KAT expressions $p_{1}, p_{2} \in K$ with

$$
\llbracket p_{j} \rrbracket=\sum_{i=0}^{\infty} \sum_{\sigma:\{1, \ldots, i\} \rightarrow\{1, \ldots, k\}} b_{\sigma(1) \ldots \sigma(i)}^{(j)} p_{\sigma(1)} \ldots p_{\sigma(i)}
$$

we define $p_{1} \leq p_{2}$ iff $p_{1}=p_{2}$ or there exist some sequence $\alpha$ such that $v\left(b_{\alpha}^{(1)}\right)<$ $v\left(b_{\alpha}^{(2)}\right)$ and $v\left(b_{\beta}^{(1)}\right)=v\left(b_{\beta}^{(2)}\right)$ hold for all $\beta<\alpha$.

For instance, we could write $b \in B$ as a disjunction of minterms over $P$, and use this to define $v(b)$ using the equations

$$
v(b)=\frac{1}{2}=v(\bar{b}) \text { for } b \in P, \quad v\left(b_{1} b_{2}\right)=v\left(b_{1}\right) \cdot v\left(b_{2}\right), \quad v\left(b_{1}+b_{2}\right)=v\left(b_{1}\right)+v\left(b_{2}\right)
$$

The fps order defined by this valuation guarantees that all rewrite rules obtained from the preference equations above will have the desired property. More generally, we can now characterise some classes of rewrite rules that are of particular interest by the property that we obtain a descending sequence of KAT expressions with respect to some fps order.

Definition 5 A set $\mathcal{R}$ of rewrite rules $\ell \rightsquigarrow r$ derived from a set $\Sigma$ of equations $\ell=r$ on $K$ is safe iff there exists an fps order $\leq$ on $K$ such that $r \leq \ell$ holds for all rewrite rules in $\ell \rightsquigarrow r \in \mathcal{R}$, and for all rewrite rules in $\ell \rightsquigarrow r \in \mathcal{R}$ the right hand side $r$ is a polynomial. 
Note that we do not require $r<\ell$, because we can only have finitely many equivalent terms. The following proposition states that indeed a safe set of rewrite rules will guarantee termination.

Proposition 3 If $\mathcal{R}$ is a safe set of rewrite rules on $K$, then term rewriting with $\mathcal{R}$ will always terminate.

Proof As we have $r \leq \ell$ for all rewrite rules $\ell \rightsquigarrow r$ term rewriting produces a decreasing sequence of expressions $p_{0}, p_{1}, p_{2}, \ldots$ There cannot be infinitely many $p_{i}$ with the same valuation, so the rewriting terminates with an expression of minimal valuation.

Note that our definition of fps-order does not require the full expansion of the power series representation, which would be infeasible. Furthermore, the termination result assumes that we apply rewrite rules to finite expressions, not to the expanded power series. The restriction that right hand sides of rewrite rules must be polynomials could thus be relaxed by the requirement that rules not satisfying this property can only be applied finitely many times.

\subsection{Church Rosser property}

Besides termination we are interested in a unique result of the rewriting process, for which we have to investigate confluence. Formally, (conditional) term rewriting defines a binary relation $\rightsquigarrow$ on terms. Let $\rightsquigarrow *$ denote its reflexive, transitive closure.

Definition 6 A (conditional) term rewriting system is confluent iff for all terms $t, t_{1}$, $t_{2}$ whenever $t \rightsquigarrow^{*} t_{1}$ and $t \rightsquigarrow^{*} t_{2}$ hold, there exists a term $t^{\prime}$ with $t_{1} \rightsquigarrow^{*} t^{\prime}$ and $t_{2} \rightsquigarrow^{*} t^{\prime}$.

A (conditional) term rewriting system is locally confluent iff for all terms $t, t_{1}, t_{2}$ whenever $t \rightsquigarrow t_{1}$ and $t \rightsquigarrow t_{2}$ hold, there exists a term $t^{\prime}$ with $t_{1} \rightsquigarrow * t^{\prime}$ and $t_{2} \rightsquigarrow * t^{\prime}$.

A terminating and confluent (conditional) term rewriting system satisfies the Church-Rosser property, i.e. for each term $t$ there exists a unique term $t^{*}$ with $t \rightsquigarrow{ }^{*} t^{*}$ such that no rewrite rule is applicable to $t^{*}$. The term $t^{*}$ is usually called the normal form of $t$. In order context of WIS personalisation, in which $t$ and $t^{*}$ would represent plots, we call $t^{*}$ the personalisation of $t$ with respect to the preference rules used for term rewriting.

For terminating term rewriting systems the notions of confluence and local confluence coincide. Therefore, assuming that we use an fps-order we may restrict our attention to local confluence. Nevertheless, it is easy to see that the application of the rewrite rules as defined in the previous section will not be locally confluent. In order to circumvent this problem we suggest to adopt critical pair completion from the approach of Knuth and Bendix [12].

Definition 7 In case $t \rightsquigarrow^{*} t_{1}$ and $t \rightsquigarrow^{*} t_{2}$ hold and neither $t_{1}$ nor $t_{2}$ permit a further application of rewrite rules, the pair $\left(t_{1}, t_{2}\right)$ is called critical.

The idea of critical pair completion is to add a new rewrite rule $t_{1} \rightsquigarrow t_{2}$ (and its reverse) and continue the rewriting process. In the presence of an fps-order, however, 
we would check first if $t_{1} \leq t_{2}$ holds or $t_{2} \leq t_{1}$. In the former case we would add $t_{2} \rightsquigarrow t_{1}$, in the latter one $t_{1} \rightsquigarrow t_{2}$ to the set of rewrite rules. Obviously, in this way we enforce confluence and thus the Church-Rosser property.

Proposition 4 If a safe set $\mathcal{R}$ of rewrite rules on $K$ is combined with critical pair completion, term rewriting with $\mathcal{R}$ will satisfy the Church-Rosser property.

Proof Due to Proposition 3 term rewriting with $\mathcal{R}$ will terminate. If there is more than one possible way to terminate, we will have a critical pair and thus be able to continue the rewriting process with an extended set of rules. Due to the wellfoundedness of the fps-order the process cannot continue forever, as we either terminate with 0 or with a unique minimal expression.

Example 3 In Example 2 we actually applied the rewrite step $\alpha_{3} \rightsquigarrow\left(\varphi_{1}+\bar{\varphi}_{1}\right) \alpha_{3}$. This can be rewritten to $\varphi_{1} \alpha_{3}+\overline{\varphi_{1}} \alpha_{3}$ and further to $\bar{\varphi}_{1} \alpha_{3}$ using the preference rule $\varphi_{1} \alpha_{3}=0$. On the other hand we could undo the introduction of the disjunction and rewrite the term to $\alpha_{3}$. Critical pair completion would thus add the rewrite rule $\alpha_{3} \rightsquigarrow \overline{\varphi_{1}} \alpha_{3}$ and thus enforce the Church-Rosser property.

\subsection{Compatibility of preference rules with deontic constraints}

In the introduction we explained that the concept of actor in storyboarding does not only refer to user profiles and associated preferences but also to user roles and associated obligations and rights. For these we can use a propositional deontic logic [22]. Now the question arises how to coordinate the deontic constraints of a role with preferences of a user in that role (according to some profile).

Let us first introduce the logical language $\mathcal{L}$ for obligations and rights.

Definition 8 The set of deontic formulae is the smallest set $\mathcal{L}$ with the following properties:

- All propositional atoms are also atoms of $\mathcal{L}$.

- If $\alpha$ is an action on scene $s$ and $r$ is a role associated with $s$, then $\mathbf{O} d o(r, \alpha)$ is an atom of $\mathcal{L}$.

- If $\alpha$ is an action on scene $s$ and $r$ is a role associated with $s$, then $\mathbf{P} d o(r, \alpha)$ is an atom of $\mathcal{L}$.

- If $\alpha$ is an action on scene $s$ and $r$ is a role associated with $s$, then $\mathbf{F} d o(r, \alpha)$ is an atom of $\mathcal{L}$.

- If $\alpha$ is an action on scene $s$ and $r$ is a role associated with $s$, then $d o(r, \alpha)$ is an atom of $\mathcal{L}$.

- For $\varphi, \psi \in \mathcal{L}$ we also have $\neg \varphi, \varphi \wedge \psi, \varphi \vee \psi, \varphi \Rightarrow \psi$ and $\varphi \Leftrightarrow \psi$ are also formulae in $\mathcal{L}$.

The informal meaning of the operators $\mathbf{O}, \mathbf{P}$ and $\mathbf{F}$ is standard: $\mathbf{O} d o(r, \alpha)$ means that an actor with role $r$ is obliged to perform action $\alpha, \mathbf{P} d o(r, \alpha)$ means that an actor with role $r$ is permitted to perform action $\alpha$, and $\mathbf{F} d o(r, \alpha)$ means that an actor with role $r$ is forbidden to perform action $\alpha$. The meaning of the atom $\operatorname{do}(r, \alpha)$ is that an actor in role $r$ will actually perform action $\alpha$. 
Example 4 Recall from our simple Example 1 that $\alpha_{2}$ is the payment_by_card action, and $\alpha_{4}$ is the payment_by_cheque action. The condition $\varphi_{1}=$ price_in_range expresses that the price of the selected product lies within a range permitted for credit card payment. Then the deontic formula

$$
\left.\neg \varphi_{1} \Rightarrow \mathbf{F} \text { do (new_customer, } \alpha_{4}\right)
$$

expresses that if the price of the selected product is outside the specified range for credit card payment, a new customer may also not pay by cheque. The deontic constraint

$$
\left.\left.\mathbf{P} \text { do (trusted_customer, } \alpha_{2}\right) \wedge \mathbf{P} d o \text { (trusted_customer, } \alpha_{4}\right)
$$

expresses that a trusted customer is allowed to unconditionally pay by credit card or cheque.

Let us briefly discuss the semantics of our logic, for which we use status sets.

Definition 9 A status set of $\mathcal{L}$ is a set $\mathcal{S}$ of atoms satisfying $\mathbf{P} d o(r, \alpha) \in \mathcal{S}$ iff F $d o(r, \alpha) \notin \mathcal{S}$.

If $\Sigma$ is a set of formulae in $\mathcal{L}$, then a status set $\mathcal{S}$ is feasible iff it satisfies $\Sigma$ in the usual propositional sense, i.e. $\mathcal{S}$ determines the truth values of the atoms, and the usual interpretation for negation, conjunction and disjunction applies. $\mathcal{S}$ is closed iff we have $\mathbf{O} d o(r, \alpha) \in \mathcal{S} \Rightarrow d o(r, \alpha) \in \mathcal{S}$ and $d o(r, \alpha) \in \mathcal{S} \Rightarrow \mathbf{P} d o(r, \alpha) \in \mathcal{S}$.

Obviously, we can use the rules defining closed status sets to build the closure of any status set.

Definition 10 A sequence $\mathcal{S}_{0} \rightarrow \mathcal{S}_{1} \rightarrow \cdots \rightarrow \mathcal{S}_{n}$ is enabled iff all $\mathcal{S}_{i}$ are closed and feasible with respect to $\Sigma$, and for each $i=0, \ldots, n-1$ there is some action $\alpha_{i}$ and some role $r$ with $d o\left(r, \alpha_{i}\right) \in \mathcal{S}_{i}$ such that the execution of $\alpha_{i}$ will produce the status set $\mathcal{S}_{i+1}$ and the sequence of actions $\alpha_{0}, \alpha_{1}, \ldots, \alpha_{n}$ is permitted by the story space.

Example 5 Take the actions and Boolean conditions in Example 1 and let $\Sigma$ be the set of deontic constraints in Example 4. Let $r$ denote the role new_customer. Then the following sequence of status sets is enabled:

$$
\begin{aligned}
& \mathcal{S}_{0}=\left\{d o\left(r, \alpha_{1}\right), \mathbf{P} \operatorname{do}\left(r, \alpha_{1}\right)\right\} \cup\left\{\mathbf{F} \operatorname{do}\left(r, \alpha_{i}\right) \mid i \neq 1\right\} \\
& \mathcal{S}_{1}=\left\{\neg \varphi_{1}, \operatorname{do}\left(r, \alpha_{3}\right), \mathbf{P} \operatorname{do}\left(r, \alpha_{3}\right)\right\} \cup\left\{\mathbf{F} \operatorname{do}\left(r, \alpha_{i}\right) \mid i \neq 3\right\} \\
& \mathcal{S}_{2}=\left\{\varphi_{3}, \operatorname{do}\left(r, \alpha_{5}\right), \mathbf{P} \operatorname{do}\left(r, \alpha_{5}\right)\right\} \cup\left\{\mathbf{F} \operatorname{do}\left(r, \alpha_{i}\right) \mid i \neq 5\right\} \\
& \mathcal{S}_{3}=\left\{\varphi_{5}, \operatorname{do}\left(r, \alpha_{6}\right), \mathbf{P} \operatorname{do}\left(r, \alpha_{6}\right)\right\} \cup\left\{\mathbf{F} \operatorname{do}\left(r, \alpha_{i}\right) \mid i \neq 6\right\} \\
& \mathcal{S}_{4}=\left\{\mathbf{P} \operatorname{do}\left(r, \alpha_{1}\right)\right\} \cup\left\{\mathbf{F} \operatorname{do}\left(r, \alpha_{i}\right) \mid i \neq 1\right\}
\end{aligned}
$$

The corresponding sequence of actions is $\alpha_{1} \alpha_{3} \alpha_{5} \alpha_{6}$.

Example 5 suggest that not all sequences of actions that are permitted by the plot are compatible with deontic constraints. This rises the question, whether the plot can 
be rewritten in a way that all stories it defines correspond to an enabled sequence of status sets.

For this we consider only deontic constraints associated with a single role. If $\Sigma$ is such a set of deontic constraints, we can assume that each formula $\varphi \in \Sigma$ is written as a disjunction of literals, i.e. atoms or negated atoms. If this were not the case, we can transform $\varphi$ into conjunctive normal form, say $\varphi \equiv \varphi_{1} \wedge \cdots \wedge \varphi_{n}$, and replace $\varphi$ by $\varphi_{1}, \ldots, \varphi_{n}$. This does not change the semantics, as satisfaction of a conjunction of constraints is equivalent to satisfaction of all conjuncts.

For $\varphi \in \Sigma$ define $\mathcal{P}_{\varphi}$ be the set of Boolean atoms $\psi$ with $\neg \psi$ occurring in $\varphi$, negated Boolean atoms $\bar{\psi}$ with $\psi$ occurring in $\varphi$, and actions $\alpha$ such that one of $\mathbf{F} d o(r, \alpha), \neg \mathbf{P} d o(r, \alpha), \neg \mathbf{O} d o(r, \alpha)$ or $\neg d o(r, \alpha)$ occurs in $\varphi$. Let $\mathcal{C}_{\varphi}$ be the set of actions $\beta$ such that $\mathbf{O} d o(r, \alpha)$ or $\operatorname{do}(r, \alpha)$ occurs in $\varphi$. Let $\mathcal{P}_{\varphi}=\left\{p_{1}, \ldots, p_{k}\right\}$ and $\mathcal{C}_{\varphi}=\left\{q_{1}, \ldots, q_{m}\right\}$.

Then each permutation $\sigma$ of $\{1, \ldots, k\}$ gives rise to an equation

$$
p_{\sigma(1)} \ldots p_{\sigma(k)}=\sum_{j=1}^{m} p_{\sigma(1)} \ldots p_{\sigma(k)} q_{j}
$$

which degenerates to $p_{\sigma(1)} \ldots p_{\sigma(k)}=0$ in case $\mathcal{C}_{\varphi}=\emptyset$.

Example 6 If $\varphi$ is $\varphi_{1} \vee \neg \varphi_{2} \vee \neg d o(r, \alpha)$, then we obtain $\mathcal{P}_{\varphi}=\left\{\bar{\varphi}_{1}, \varphi_{2}, \alpha\right\}$, which leads (among others) to the equation $\bar{\varphi}_{1} \varphi_{2} \alpha=0$. In fact, the deontic constraint can be written as $\neg\left(\neg \varphi_{1} \wedge \varphi_{2} \wedge d o(r, \alpha)\right)$, i.e. it is forbidden to have at the same time $\neg \varphi_{1}$ and $\varphi_{2}$ and execute $\alpha$, which is what the equation expresses.

Similarly, for the first constraint in Example 4 we obtain $\mathcal{P}=\left\{\bar{\varphi}_{1}, \alpha_{4}\right\}$ leading to the equation $\bar{\varphi}_{1} \alpha_{4}=0$, which is a natural consequence: if $\varphi_{1}$ is not satisfied, $\alpha_{4}$ is not permitted.

The other two constraints in Example 4, however, lead to $\mathcal{P}=\mathcal{C}=\emptyset$, which does not define any equation. Naturally, the constraint expresses a right to execute $\alpha_{2}$ and $\alpha_{4}$, which does not press any restrictions on the plot.

Similarly, the constraint $\varphi \equiv \varphi_{1} \vee \neg \mathbf{P} d o\left(r, \alpha_{1}\right) \vee \mathbf{O} d o\left(r, \alpha_{2}\right)$ leads to $\mathcal{P}=\left\{\bar{\varphi}_{1}, \alpha_{1}\right\}$ and $\mathcal{C}=\left\{\alpha_{2}\right\}$, which defines the equation $\bar{\varphi}_{1} \alpha_{1}=\bar{\varphi}_{1} \alpha_{1} \alpha_{2}$, i.e. if $\varphi_{1}$ does not hold and $\alpha_{1}$ is executed, then also $\alpha_{2}$ must be executed.

Thus, each set $\Sigma$ of deontic constraints defines a set $E$ of equations on the plot. In contrast to the preference rules, however, the equations in $E$ do not just express preferences, but strict restrictions. Technically speaking, if we can rewrite the plot $p$ to a plot $p^{\prime}$ using the equations in $E$, then each story enabled by $p^{\prime}$, i.e. a sequence of actions, corresponds to a sequence of status sets that is enabled by $\Sigma$. We can then apply term rewriting with respect to preference rules to the plot $p^{\prime}$.

\section{Fine tuning using parameterised plots}

In this section we want to generalise our approach to WIS customisation by taking content into consideration. In the previous section we concentrated on the usage level, i.e. a very high-level of abstraction, for which the use of KATs turned out to be a very useful foundation. However, the user actions appearing in a plot are left 
totally unspecified. The only properties we can associate with them are propositional pre- and postconditions.

On a lower level of abstraction, the conceptual layer in [22], the elementary scenes of the story space are associated with so-called media types, which combine a view on some underlying database with operations that update the underlying database and manipulate views, and some other mechanisms such as cohesion preorders and hierarchical versions that support adaptivity and granularity. Consequently, the actions appearing in the plot will be refined by operations associated with some media type; they will become parameterised and specified by an operation body. Furthermore, the Boolean conditions can be replaced by at least first-order conditions that can be evaluated against the underlying database.

That is, on the conceptual level we obtain a complex specification of operations and conditions, which will require a different approach to the customisation problem. In general, we can define a multi-modal logic as in [19], in which customisation could be expressed as a proof obligation. However, this logic would be higher-order and customisation would therefore be undecidable. Carrying over our approach by term rewriting would have little chances to guarantee the Church-Rosser property. Therefore, we first investigate a simplified approach considering only the parameters of operations and first-order conditions, which will lead us to parameterised plots.

\subsection{Media types}

A media type is an abstraction of content and functionality. The name refers to the web as a medium for mass communication, and types for classification and formal abstraction from such communication means. At its core a media type has a view on some underlying database schema. Furthermore, the defining query for the view should create identifiers (or abstract URIs) that permit to create interlinked sets of media objects, i.e. instances of media types.

So, let $\mathcal{S}$ denote some conceptual database schema. The introduction of media types in [22] was based on the higher-order Entity-Relationship model (HERM) [25], but the choice of data model is of minor importance. A model (such as HERM) with richer structuring mechanisms will simplify the definition of views, while a poorer model-even if it has the same expressive power-will require more sophistication in the queries that define views.

Furthermore we assume a type system comprising base types and various type constructors, e.g.

$$
t=b|\mathbb{1}|\left(a_{1}: t_{1}, \ldots, a_{n}: t_{n}\right)|\{t\}|[t]|\langle t\rangle|\left(a_{1}: t_{1}\right) \oplus \cdots \oplus\left(a_{n}: t_{n}\right)
$$

would define a type system with not further specified base types $b$, a trivial type 11 , and constructors for records, finite sets, lists and multisets, and disjoint unions. Usually, we assume that among the base types there is a type URI representing abstract surrogates for URIs.

The semantics of such types is primarily defined by their domains, i.e. with each type $t$ we associate a set of values $\operatorname{dom}(t)$. For a base type the domain is assumed to be countably infinite. For the trivial type the domain is a singleton set $\{\mathbf{1}\}$. For constructed types we use the usual recursive definitions. Furthermore, we associate basic (fixed) operations with the type system, e.g. projection on record types, union for sets, etc., but we dispense with listing them here. 
The type system forms the basis for the definition of content type expressions. We simply extend the type system permitting $r: M$ to appear in lieu of a base type, with $r$ and $M$ being names for a reference and a media type, respectively, both taken from some unspecified countable pool of names. Replacing $r: M$ by $U R I$ results in a proper type, called content representation type.

\section{Definition 11 A media type $M$ consists of}

- a content type expression $\operatorname{ct}(M)$ with corresponding content representation type $t_{M}$

- $\quad$ a query $q_{M}$ defined on $\mathcal{S}$ with output type (id: URI, value $: t_{M}$ ),

- a cohesion preorder $\preceq$ or a set of cohesion proximity values,

- a set $\mathcal{H}(M)$ of hierarchical versions, and

- a finite set $O p_{M}$ of operations.

An media schema $\mathcal{M}$ is a finite set of media types that is closed under references, i.e. whenever $r: M$ appears in a content type expression $\operatorname{ct}\left(M^{\prime}\right)$ with $M^{\prime} \in \mathcal{M}$, then $M \in \mathcal{M}$ must also hold.

In the following we will define queries and operations, but we will dispense with a definition of the other extensions, as they are not relevant for the customisation problem. Cohesion and hierarchical versions have been discussed in detail in [22].

In general, a query $q$ consists of an input database schema $\operatorname{in}(q)$, an output database schema $\operatorname{out}(q)$, and a query mapping $\hat{q}: \operatorname{inst}(\operatorname{in}(q)) \rightarrow \operatorname{inst}(\operatorname{out}(q))$, which maps instances (i.e. databases) over the input database schema to instances over the output database schema. In our case we have $\operatorname{in}(q)=\mathcal{S}$, i.e. for all queries the input schema is the underlying database schema of the WIS. The output schema contains a single type not appearing in $\mathcal{S}$ - this type has been specified in Definition 11 above. Then the query mapping $\hat{q}$ will transform a database $d b$ over $\mathcal{S}$ into a set of pairs $(u, v)$ with abstract URIs $u$ and values $v$ of type $t_{M}$. As discussed in [22] such queries are best expressed by languages that will actually create more than just values of the given type, i.e. both input and output schema are subschemata of a larger "working schema".

Let us briefly discuss the semantics of media types and schemata. Taking an database $d b$ over the database schema $\mathcal{S}$ and execute the queries $q_{M}$ for all $M \in \mathcal{M}$ will result in sets $d b(M)$ of media objects, i.e. values $(u, v)$ with a URI $u$ and a value $v$ of type $t_{M}$. We request that whenever a URI $u^{\prime}$ appear in such a value corresponding to the reference $r: M^{\prime}$ in the content type expression $\operatorname{ct}(M)$, then $d b\left(M^{\prime}\right)$ must contain a value $\left(u^{\prime}, v^{\prime}\right)$. Furthermore, URIs must be globally unique.

In this way we obtain an abstraction from the structure at the interface. The value $(u, v) \in d b(M)$ represents the content $v$ at the abstract URI $u$ including references to other units of content, i.e. including the navigation structure. As emphasised in [22] and discussed with respect to different query languages, the abstract definition of media types requires the use of query languages that are able to create abstract identifiers, i.e. the URIs, and in many cases will need a fixed-point construction.

Operations associated with an media type permit updating the underlying database and to open and close media objects. For this, we require a signature describing input- and output-parameters as well as a selection type, and an operation body. The selection type specifies, which part of the content value must be selected as a 
precondition for the operation being executed. This form of interaction types captures form processing in a natural way and on a reasonably high level of abstraction, but it is not limited to this. The operation body can be specified using the following constructs:

- The operations skip and fail do not change anything or are not defined, respectively.

- An assignment $f\left(t_{1}, \ldots, t_{n}\right):=\exp$ with an updatable function $f$ in the signature of arity $n$ and an expression of the same type as $x$ is an operation. Here we permit expressions $\mathbf{I} x . \varphi$ with the meaning of "the unique $x$ satisfying $\varphi$ ". In particular, $\varphi$ can be used to express the result of a query.

- If $p, p_{1}$ and $p_{2}$ are operations, the same holds for the iteration $p^{*}$, the choice $p_{1} \square p_{2}$, the sequence $p_{1} ; p_{2}$, and the parallel block $p_{1} \| p_{2}$.

- If $p$ is an operation and $\varphi$ is a condition, then the guarded program $\{\varphi\} p$ and the postguarded program $p\{\varphi\}$ are also operations.

- If $x$ is a variable and $p$ is an operation, then the selection @ $x\{\varphi\} \bullet p$-choose some $x$ satisfying $\varphi$ and execute $p$ with this $x$-is also an operation.

Example 7 Let us look at the scene $s_{0}=$ product in Example 1, which has to be supported by a media type $M_{0}=$ Product_List. First we have to define the content type expression

$$
\begin{aligned}
\operatorname{ct}\left(M_{0}\right)= & {\left[\left(\mathrm{p} \_\mathrm{id}: I D, \text { description }: \operatorname{STRING}, \text { price }:\right.\right. \text { DECIMAL, }} \\
& \text { details : Product_DetaIls, amount : }(\text { sel }: \text { CARD }) \oplus(\text { empty }: \mathbb{1}))]
\end{aligned}
$$

That is, a user will be presented with a list of products, each with a product-id, a description, which is meant to be just a descriptive product name, its price, a link to further details and the selected number of items to be purchased-this will be initially undefined.

The corresponding content representation type will be

$$
\begin{aligned}
t_{M_{0}}= & (\text { p_id : ID, description : STRING, price : DECIMAL, } \\
& \text { details : } U R I, \text { amount : }(\text { sel }: C A R D) \oplus(\text { empty }: \mathbb{1}))]
\end{aligned}
$$

In order to build media objects of type $M_{0}$ we need some information about the underlying database schema. Let us assume we have at least four relation types ProDUCT with attributes p_id, description, category and price, REvIEW with attributes p_id, number, date and text, SPECIAL_OfFer with attributes o_id, start_date, end_date, conditions and discount, and IN_OFFER with attributes p_id, o_id, minimum.

On top of this database schema we have to define the defining query $q_{M_{0}}$. As we have to create links to product details, we will also need the media type $M_{0}^{\prime}=$ Product_Details. For this we define the content type expression

$$
\begin{aligned}
\operatorname{ct}\left(M_{0}^{\prime}\right)= & \left(\mathrm { p } \_ \mathrm { id } : I D , \text { category } : \text { STRING } , \text { offers : } \left\{\left(\mathrm{o} \_\mathrm{id}: I D, \text { conditions : STRING },\right.\right.\right. \\
& \text { minimum }: C A R D, \text { discount }: \text { DECIMAL })\}, \text { reviews : }\{(\text { text }: \text { STRING })\})
\end{aligned}
$$

which does not contain references and thus coincides with its corresponding content representation type $t_{M_{0}^{\prime}}$. According to [22] we can use an IQl-like query language to 
define $q_{M_{0}}$. In doing so, we build a sequence of logic programs. The first block simply contains a single rule

$$
\operatorname{PLIST}\left(u_{-} p l, P\right) \text {. }
$$

This will create a new relation with a single entry, which contains a single pair of URIs. The first URI will be the abstract URI for our product list; the second one is a temporary surrogate for the list data. The second logic program collects the list entries. Again, we only have a single rule

$$
\hat{P}\left(p, d, p r, u_{-} p d, \mathbf{1}\right) \leftarrow \operatorname{Product}(p, d, c, p r) .
$$

So we create a relation corresponding to the URI $P$ created by the first program. Each tuple in that relation contains a product identifier, the corresponding product description and price, the constant 1 of type 11 (nothing has been selected), and a freshly created URI $u_{-} p d$ representing the link to product details.

The next logic program creates product details. It also consists of a single rule

$$
\widehat{u_{-} p d}(p, c, O, R) \leftarrow \hat{P}\left(p, d, p r, u_{-} p d, x\right), \operatorname{Product}(p, d, c, p r) .
$$

with new surrogate URIs $O$ and $R$ representing offers and reviews, respectively. The corresponding relations $\hat{O}$ and $\hat{R}$ are defined by the next logic program:

$$
\begin{aligned}
\hat{O}\left(o, c^{\prime}, m, d\right) \leftarrow & \widehat{u_{-} p d}(p, c, O, R), \operatorname{In} \_\operatorname{Offer}(p, o, m), \\
& \quad \operatorname{Special} \_\operatorname{Offer}\left(o, s, e, c^{\prime}, d\right), s \leq \text { now } \leq e . \\
\hat{R}(t) \leftarrow & \widehat{u_{-} p d}(p, c, O, R), \operatorname{Review}(p, n, d, t) .
\end{aligned}
$$

Then the following program with again only one rule substitutes the actual values for the surrogate URIs:

$$
\overline{u_{-} p d}(p, c, \hat{O}, \hat{R}) \leftarrow \widehat{u_{-} p d}(p, c, O, R) .
$$

The last logic program in the sequence actually creates the media objects replacing the surrogate URIs in PLIST:

Product_List $\left(u \_p l, P L\right) \leftarrow \operatorname{PLIST}\left(u_{-} p l, P\right), P L=$ list_of $(\hat{P})$ order_by 1 ;

Product_Details $\left(u_{-} p d, \overline{u_{-} p d}\right) \leftarrow \operatorname{Plist}\left(u_{-} p l, P\right), \hat{P}\left(p, d, p r, u_{-} p d, x\right)$.

Let us now look at the action $\alpha_{1}=$ select_product from Example 1. Operations on media types abstract from low-level user interface aspects. So the fact that a user enters values for the number of items of some selected products (including iterations in case of entry errors) is simply reflected in a selection type

$$
\operatorname{sel}_{1}=\left[\left(\mathrm{p} \_\mathrm{id}: I D, \text { price }: D E C I M A L, \text { amount }: C A R D\right)\right] \text {. }
$$

All products that are not selected (or the amount value is set to 0) will not appear in the value $\mathrm{sel}$ of type $\mathrm{sel}_{1}$. There are no other input parameters, and for simplicity we also dispense with output, so the only effect the action will have is to place an 
order into the database, close the product selection view, i.e. close the media object of type Product_List, and open a media object of type PAYMENT.

So, let us assume the database schema contains at least two more relations ORDER with attributes order_id, price and status, and IN_ORDER with attributes order_id, p_id and amount. Due to the use of special offers the sum of the prices of the ordered products may differ from the total price of the order. Then the core of the operation refining $\alpha_{1}$ is defined by the fragment

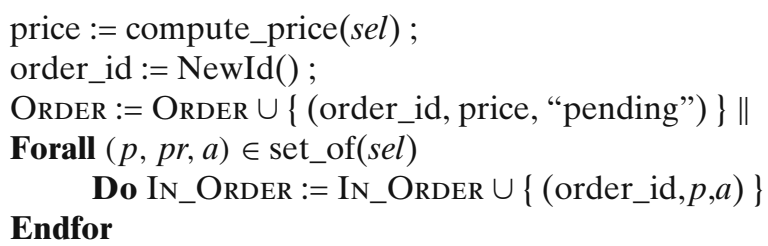

We dispense with the details of the procedure compute_price, which would have to select the minimum price with items grouped according to special offers.

Note that Example 7 is still rather simplistic. In fact, we would like to refine product selection permitting iteration, restriction of the product list, switching between descriptions and product list, etc. The transition to the next scene would only occur after confirming the selection. However, for illustration of the concept of media type this simple example should suffice.

\subsection{A modal logic for reasoning about WIS specifications}

Before looking at a generalisation of the term rewriting approach let us take a brief look into a general approach to preference customisation with respect to media types. Using modal operators $[p]$ and $\langle p\rangle$ we can extend the logic $\mathcal{L}$ that we use for conditions on databases.

Definition 12 Let $\mathcal{M}$ be a media schema on top of a database schema $\mathcal{S}$, and let $\mathbb{F}$ denote the formulae of a logic $\mathcal{L}$ associated with $\mathcal{S}$, i.e. formulae in $\mathbb{F}$ can be evaluated in databases $d b$ over $\mathcal{S}$. Then $\mathbb{F}_{\mathcal{M}}$ denotes the smallest set of formulae that contains $\mathbb{F}$, is closed under conjunction, disjunction, negation, and existential quantification, and contains all formulae of the form $[p] \varphi$ with an operation $p$ defined on some media type $M \in \mathcal{M}$ and a formula $\varphi \in \mathbb{F}_{\mathcal{M}}$. This set is the set of formulae of the logic of media types $\mathcal{L}_{\mathcal{M}}$ on $\mathcal{M}$.

The meaning of $[p] \varphi$ is that after execution of $p$ the formula $\varphi$ will necessarily hold, no matter, which choice was made during the execution. We can use the logic $\mathcal{L}_{\mathcal{M}}$ to formulate a "proof obligation" for customisation [19].

As usual in modal logic we use $\langle p\rangle \varphi$ as a shortcut for $\neg[p] \neg \varphi$ meaning that after the execution of $p$ the formula $\varphi$ will possibly hold, i.e. there is a sequence of choices under which $\varphi$ will hold. Furthermore, the following rules can be applied to eliminate modal operators in formulae:

- $\quad[$ skip $] \varphi \equiv \varphi$

$-[\mathrm{fail}] \varphi \equiv$ false,

- $\quad[x:=t] \varphi \equiv \varphi\{x / t\}$, i.e. substitute $t$ for all free occurrences of $x$ in $\varphi$, 
- $\quad\left[p_{1} \square p_{2}\right] \varphi \equiv\left[p_{1}\right] \varphi \wedge\left[p_{2}\right] \varphi$,

- $\left[p_{1} ; p_{2}\right] \varphi \equiv\left[p_{1}\right]\left[p_{2}\right] \varphi$,

- $\left[p^{*}\right] \varphi$ is the weakest solution $\chi$ of $\chi \leftrightarrow \varphi \wedge[p] \chi$,

- $\quad[\{\psi\} p] \varphi \equiv \psi \rightarrow[p] \varphi$,

- $\quad[p\{\psi\}] \varphi \equiv[p](\psi \rightarrow \varphi)$, and

- $[@ x\{\psi\} \bullet p] \varphi \equiv \forall x .(\psi \rightarrow[p] \varphi)$.

Note that this modality elimination is only partial, as the parallel block operator cannot be eliminated this way. In order to obtain also a rule for the parallel block we have to break parallel composition down to "proclets" [2], i.e. elementary blocks that do not contain any parallelism. Furthermore, the rule for iteration involves finding a weakest solution.

Also note that the logic is intrinsically higher-order, even if the logic $\mathcal{L}$ was firstorder. This results from the assignment rule in case the term represents the result of a query. As queries involve a fixed-point constructor, the formula resulting from this substitution will be of a higher order.

Therefore, approaching customisation to preferences on the basis of the multimodal logic of media types will require much deeper logical treatment, which we cannot handle here, let aside the fact that little is known about higher-order dynamic logics.

\subsection{Parametrised plots and preference rules}

In order to simplify the approach to customisation on the basis of media types we now introduce parameterised plots. That is, we replace the atomic actions by operations with parameters blurring the distiction between input parameters and the selection parameter, but ignoring the operation body. Similarly, we replace the Boolean conditions by formulae that can be evaluated on the basis of the parameters in the operations and the underlying database.

Example 8 Let us take the plot from Example 1. As seen in Example 7 the action $\alpha_{1}$ has to take a parameter sel of type

$$
s e l_{1}=\left[\left(\mathrm{p} \_ \text {id }: I D, \text { price }: D E C I M A L, \text { amount }: C A R D\right)\right] .
$$

Similarly, $\alpha_{2}$ (payment_by_card) takes parameters card_no, expiry, card_holder and amount, $\alpha_{3}$ (payment_by_bank_transfer) takes account_no, bank_code, account_name and amount, and $\alpha_{4}$ (payment_by_cheque) takes billing_name and amount. Action $\alpha_{5}$ (enter_address) takes a parameter, which is either customer_id or billing_address, while $\alpha_{6}$ (confirm_order) takes no parameter. We omit the obvious types associated with these parameters.

The condition $\varphi_{1}$ becomes total_price $($ sel $) \leq \ell$, where the total price is determined by the procedure compute_price() in Example 7, and $\ell$ is a constant. Conditions $\varphi_{i}$ with $i \in\{2,3,4\}$ become payment_method(card), payment_method(bank) and payment_method(cheque), respectively, and $\varphi_{5}$ turns into confirmed(order_id).

Without loss of generality we may restrict ourselves to finite domains. That is, we will only consider cardinals, integers, decimals, etc. within certain bounds, which 
nonetheless can be arbitrarily large, character strings and lists will have a maximum length, and also the cardinality of sets will be bound by a maximum. Then a parameterised operation actually represents the finite choice of all its instantiations, and a condition represents the finite disjunction of all its instantiations-of course only bound variables will be instantiated. Consequently, a parameterised plot is still a KAT expression, only that the carrier set is significantly enlarged.

In the same spirit as in Section 2 we may introduce preference rules. Roughly speaking, the parameterised plot represents a large collection of choices, and a preference rule reduces these choices. Formally, in line with Section 2 and the previous subsection on modal logic we can rewrite a parametrised plot by a formal power series with logical coefficients and sequences of parametrised operations as monoms. If we make the further assumption that there is a (large) bound on the number of iterations we even obtain polynomials.

Definition 13 A preference rule has the form $p_{1} \rightsquigarrow p_{2}$ with bounded parametrised plots $p_{1}$ and $p_{2}$ written as polynomials

$$
p_{j}=\sum_{i=0}^{\max } \sum_{\sigma:\{1, \ldots, i\} \rightarrow\{1, \ldots, k\}} \varphi_{\sigma}^{j} p_{\sigma(1)} \ldots p_{\sigma(i)},
$$

such that for all $i$ and all $\sigma$ the coefficient $\varphi_{\sigma}^{1}$ is weaker than $\varphi_{\sigma}^{2}$, i.e. $\varphi_{\sigma}^{2} \Rightarrow \varphi_{\sigma}^{1}$ holds.

Note that in order to obtain the polynomial representation of a plot we have to shift conditions to the front, i.e. we have to replaced $p \varphi$ by $[p] \varphi p$, which amounts to reasoning with the rules of modal logic as in the previous subsection. In most cases, however, this can be avoided, as the definition only requires that conditions are strengthened, i.e. the latitude for choices will be reduced. Further note that this definition of preference already involves a direction, which we can exploit for term rewriting.

Example 9 Let us continue Examples 1 and 8, and define a few preference rules in the spirit of the preferences in [18].

Suppose a user wants to purchase a hammer and a saw with a combined price of at most 50, unless there is a special offer with at least four items including a hammer and a saw, with a discount of at least $15 \%$, and a total price not exceeding 75 .

Concentrate first on the first part of this complex preference rule. Choosing a hammer and a saw with a total price of at most 50 can be expressed by the formula

$$
\begin{aligned}
\psi_{1} & \equiv \exists p_{1}, d_{1}, p r_{1}, p_{2}, d_{2}, p r_{2} . \text { set_of }(s e l)=\left\{\left(p_{1}, p r_{1}, 1\right),\left(p_{2}, p r_{2}, 1\right)\right\} \\
& \wedge p r_{1}+p r_{2} \leq 50 \wedge \operatorname{Product}\left(p_{1}, d_{1}, \text { "hammer", } p r_{1}\right) \\
& \wedge \operatorname{Product}\left(p_{2}, d_{2}, \text { "saw", } p r_{2}\right)
\end{aligned}
$$

This would lead to the preference rule $\alpha_{1}(\mathrm{sel}) \rightsquigarrow \alpha_{1}(\mathrm{sel}) \psi_{1}$, if we ignore the second part of the preference rule starting with "unless". 
Looking only at this second part of the preference rule, we can formulate two conditions $\psi_{2}(o)$ (a special offer $o$ as specified exists) and $\psi_{3}(o)$ (the special offer $o$ has been selected) as follows:

$$
\begin{aligned}
& \psi_{2}(o) \equiv \exists s, e, c, d \text {.SPeciaL_OffFer }(o, s, e, c, d) \wedge s \leq \text { now } \leq e \wedge d \geq 15 \\
& \wedge \exists p_{1}, d_{1}, p r_{1}, p_{2}, d_{2}, p r_{2} \text {.Product }\left(p_{1}, d_{1} \text {, "hammer", } p r_{1}\right) \\
& \wedge \operatorname{Product}\left(p_{2}, d_{2} \text {, “saw", } p r_{2}\right) \\
& \wedge \exists n_{1}, n_{2} \text {.IN_OfFer }\left(p_{1}, o, n_{1}\right) \wedge \operatorname{IN} \operatorname{OFfFeR}\left(p_{2}, o, n_{2}\right) \\
& \wedge \operatorname{sum}\langle n| \exists p \text {.IN_OfFer }(p, o, n)\rangle \geq 4 \\
& \left.\wedge \frac{100-d}{100} \operatorname{sum}\langle n \cdot p r| \exists p, d, c . \text { In_Offer }(p, o, n) \wedge \operatorname{Product}(p, d, c, p r)\right\rangle \leq 75
\end{aligned}
$$

and

$$
\begin{aligned}
\psi_{3}(o) & \equiv \forall p, p r, n .(p, p r, n) \in \operatorname{set} \_ \text {of }(s e l) \leftrightarrow \exists d, c . \text { Product }(p, d, c, p r) \\
& \wedge \text { IN_Offer }(p, o, n)
\end{aligned}
$$

Then the unless statement leads to the preference rule

$$
\exists o . \psi_{2}(o) \alpha_{1}(\mathrm{sel}) \rightsquigarrow \exists o . \psi_{2}(o) \alpha_{1}(\mathrm{sel}) \exists o . \psi_{2}(o) \wedge \psi_{3}(o) .
$$

The two preference rules may conflict each other, so the first one has to be replaced by $\neg \exists o . \psi_{2}(o) \alpha_{1}(\mathrm{sel}) \rightsquigarrow \neg \exists o . \psi_{2}(o) \alpha_{1}(\mathrm{sel}) \psi_{1}$, which adequately expresses the "unless" statement.

With the specific assumptions on preference rules these give immediately rise to term rewriting rules. Furthermore, our assumptions only allow finitely many polynomials with a natural order on them as defined in Definition 13. Then the application of a preference rule in term rewriting will always decrease the parameterised plot, and due to the finiteness the process must terminate.

Proposition 5 Term rewriting on bounded parameterised plots with preference rules as defined in Definition 13 always terminates. If combined with critical pair completion, it will further satisfy the Church-Rosser property.

This result depends of course heavily on the boundedness assumption. However, this is not really a restriction, as the bounds imposed on the size of values and the number of steps in an iteration can be arbitrarily high, so that exceeding these bounds would only be possible in an untractable case.

A more severe restriction is that the application of a rewrite rule involving a condition from the logic $\mathcal{L}$ is only defined up to equivalence, and the equivalence of formulae is in general undecidable. This may force us into an interactive mode checking, whether rewriting rules can be applied or not. 


\subsection{Content customisation}

Let us finally may a first attempt widening our approach from customisation of functionality to customisation of content, which has been put aside so far. Let us start with a motivating example.

Example 10 Take the parameterised plot arising from Example 1 with the parameters discussed in Example 8. If we customize this plot using the preference rules in Example 9 the result will contain the initial segment

$$
\left(\exists o . \psi_{2}(o) \alpha_{1}(\mathrm{sel}) \exists o . \psi_{2}(o) \wedge \psi_{3}(o)\right)+\left(\neg \exists o . \psi_{2}(o) \alpha_{1}(\mathrm{sel}) \psi_{1}\right) .
$$

That is, the execution of $\alpha_{1}$ will lead to a state, in which either $\exists o . \psi_{2}(o) \wedge \psi_{3}(o)$ or $\psi_{1}$ will be satisfied. To recall, this means that either a special offer including a hammer, a saw and at least two more items, is selected, or a hammer and a saw are selected separately. If this is known, the view in Example 7 could be simplified discarding all products that will not be selected anyway. In fact, only hammers and saws and special offers associated with them have to be taken into consideration. This would lead to the following defining query:

$$
\begin{aligned}
& \operatorname{PLIST}\left(u_{-} p l, P\right) \text {; } \\
& \hat{P}\left(p, d, p r, u_{-} p d, \mathbf{1}\right) \leftarrow \operatorname{Product}(p, d \text {, "hammer", } p r) . \\
& \hat{P}\left(p, d, p r, u_{-} p d, \mathbf{1}\right) \leftarrow \operatorname{Product}(p, d, \text { "saw", pr }) . \quad ; \\
& \widehat{u_{-} p d}(p, c, O) \leftarrow \hat{P}\left(p, d, p r, u_{-} p d, x\right), \operatorname{Product}(p, d, c, p r) . \quad ; \\
& \hat{O}\left(o, c^{\prime}, m, d\right) \leftarrow \widehat{u_{-} p d}(p, c, O), \operatorname{In} \_\operatorname{Offer}(p, o, m), \\
& \operatorname{Special} \_\operatorname{Offer}\left(o, s, e, c^{\prime}, d\right), s \leq \text { now } \leq e . \quad ; \\
& \overline{u_{-} p d}(p, c, \hat{O}) \leftarrow \widehat{u_{-} p d}(p, c, O) . \quad ; \\
& \text { Product_List }\left(u \_p l, P L\right) \leftarrow \operatorname{Plist}\left(u_{-} p l, P\right), P L=\text { list_of }(\hat{P}) \text { order_by } 1 \text {; } \\
& \text { Product_Details }\left(u_{-} p d, \overline{u_{-} p d}\right) \leftarrow \operatorname{Plist}\left(u_{-} p l, P\right), \hat{P}\left(p, d, p r, u_{-} p d, x\right) \text {. }
\end{aligned}
$$

Here we used; as a sequence operator to separate the logic programs in this query.

This example raises the general question, how views can be simplified, once a customised parameterised plot is known. In the example $\psi_{1} \vee \psi_{3}$ should be used to infer a simplified view. The open research problem is to find algorithms for this. Whether this can again be achieved by term rewriting is an open problem.

\section{Conclusion}

In this paper we continued our research on the customisation of WISs according to user preferences. We approached the problem on different levels of abstraction. On a high-level of abstraction we exploit Kleene Algebras with Tests (KATs) as an algebraic approach to deal with WIS functionality customisation. This merely revises a preliminary conference publication [24]. Under the assumption of weakest 
preconditions we developed a representation of certain KAT expressions by formal power series with coefficients in a Boolean algebra, which permits an effective, terminating approach to term rewriting for certain classes of equations on KATs. When combined with critical pair completion we can actually obtain a rewrite system satisfying the Church-Rosser property. We expect that the idea can be fruitfully exploited in practice, maybe even in areas beyond web information systems.

On a lower level of abstraction we took up the idea of parameterised plots by adding parameters to the operations and replacing the Boolean conditions by firstorder formulae. However, we still did not add any details to the operations as such. On one hand this allows us to generalise the term rewriting approach, but on the other hand will still only keep or discard operations instead of simplifying them. It also offers the opportunity to widen the approach towards content customisation.

This leaves us with the more general problem of addressing WIS customisation in case we are given all details of the operations. In [24] we demonstrated that KATs are equivalent to assignment-free ASMs, while ASMs in general [3] could be used to specify the operations, as ASMs capture the notion of sequential and parallel algorithms [2,9]. The challenge is to combine our results on rewriting with ASM refinements, and to enable reasoning with dynamic logic as an approach to customisation.

There is of course one fundamental difference between ASMs in general and KATs, as besides assignments the former ones permit parallelism. As long as we do not take assignments into account, the absence of parallelism in personalisation is no loss, as in a KAT $p \| q$ can be represented as $p q+q p$, i.e. parallelism is treated merely as a choice of order. This is obviously false, if assignments are considered. A natural idea is to add a parallelism operator to KATs disregarding the fact that this could be represented by a choice of order. In order to generalise our approach to term rewriting we would need an extension to the idea of precondition. If such an extension is possible, we could still obtain a formal power series representation and hence guarantee that term rewriting will be terminating. Alternatively, we could try to shift the rewriting idea to the level of ASMs. We intend to explore both ideas in our future work.

\section{References}

1. Binemann-Zdanowicz, A., Thalheim, B.: Modeling information services on the basis of ASM semantics. In: Börger, E., Gargantini, A., Riccobene, E. (eds.) Abstract State Machines, Lecture Notes in Computer Science, vol. 2589, pp. 408-410. Springer, New York (2003)

2. Blass, A., Gurevich, J.: Abstract state machines capture parallel algorithms. ACM Trans. Comput. Log. 4(4), 578-651 (2003)

3. Börger, E., Stärk, R.: Abstract State Machines. Springer, Berlin (2003)

4. Ceri, S., Fraternali, P., Bongio, A., Brambilla, M., Comai, S., Matera, M.: Designing DataIntensive Web Applications. Morgan Kaufmann, San Francisco (2003)

5. Conallen, J.: Building Web Applications with UML. Addison-Wesley, Boston (2003)

6. Dershowitz, N.: Termination of rewriting. J. Symb. Comput. 3(1/2), 69-116 (1987)

7. De Troyer, O., Leune, C.: WSDM: a user-centered design method for web sites. In: Computer Networks and ISDN Systems-Proceedings of the 7th International WWW Conference, pp. 85-94. Elsevier, Amsterdam (1998)

8. Draheim, D., Weber, G.: Form-Oriented Analysis-A New Methodology to Model Form-Based Applications. Springer, New York (2004)

9. Gurevich, J.: Sequential abstract state machines capture sequential algorithms. ACM Trans. Comput. Log. 1(1), 77-111 (2000) 
10. Houben, G.-J., Barna, P., Frasincar, F., Vdovjak, R.: HERA: development of semantic web information systems. In: Third International Conference on Web Engineering-ICWE 2003, LNCS, vol. 2722, pp. 529-538. Springer, New York (2003)

11. Kleene, S.C.: Representation of events in nerve sets and finite automata. In: Shannon, McCarthy (eds.) Automata Studies, pp. 3-41. Princeton University Press, Princeton (1956)

12. Knuth, D.E., Bendix, P.B.: Simple word problems in universal algebras. In: Computational Problems in Abstract Algebra, pp. 263-297. Pergamon, Oxford (1970)

13. Kozen, D.: Kleene algebra with tests. ACM Trans. Program. Lang. Syst. 19(3), 427-443 (1997)

14. Kozen, D.: On Hoare logic and Kleene algebra with tests. In: Logic in Computer Science, pp. 167-172. IEEE, Piscataway (1999)

15. Kozen, D., Smith, F.: Kleene algebra with tests: completeness and decidability. In: Computer Science Logic, pp. 244-259, Utrecht, 21-27 September 1996

16. Lowe, D., Henderson-Sellers, B., Gu, A.: Web extensions to UML: using the MVC triad. In: Spaccapietra, S., March, S.T., Kambayashi, Y. (eds.) Conceptual Modeling-ER 2002, LNCS, vol. 2503, pp. 105-119. Springer, New York (2002)

17. Mayr, H.C., Schewe, K.-D., Thalheim, B., Welzer, T.: Integration of bargaining into e-business systems. Informatica 30(3), 335-345 (2006)

18. Ragone, A., Straccia, U., Di Noia, T., Di Sciascio, E., Donini, F.M.: Towards a fuzzy logic for automated multi-issue negotiation. In: Hartmann, S., Kern-Isberner, G. (eds.) Foundations of Information and Knowledge Systems (FoIKS 2008), LNCS, vol. 4932, pp. 381-396. Springer, New York (2008)

19. Schewe, K.-D.: The power of media types. In: Zhou, X., et al. (eds.) Web Information SystemsWISE 2004, LNCS, vol. 3306, pp. 53-58. Springer, New York (2004)

20. Schewe, K.-D., Thalheim, B.: Reasoning about web information systems using story algebras. In: Benczúr, A., Demetrovics, J., Gottlob, G. (eds.) Advances in Databases and Information Systems (ADBIS 2004), LNCS, vol. 3255, pp. 54-66. Springer, New York (2004)

21. Schewe, K.-D., Thalheim, B.: An algorithmic approach to high-level personalisation of web information systems. In: Fan, W., Wu, Z. Yang, J. (eds.) Web Age Information Management WAIM 2005, LNCS, vol. 3739, pp. 737-742. Springer, New York (2005)

22. Schewe, K.-D., Thalheim, B.: Conceptual modelling of web information systems. Data Knowl. Eng. 54(2), 147-188 (2005)

23. Schewe, K.-D., Thalheim, B.: Personalisation of web information systems-a term rewriting approach. Data Knowl. Eng. 62(1), 101-117 (2007)

24. Schewe, K.-D., Thalheim, B.: Term rewriting for web information systems-termination and Church Rosser property. In: Benatallah, B., et al. (eds.) Web Information Systems EngineeringWISE 2007, LNCS, vol. 4831, pp. 261-272. Springer, New York (2007)

25. Thalheim, B.: Entity-Relationship Modeling-Foundations of Database Technology. Springer, New York (2000) 